\title{
AUTONOMÍA EDUCATIVA EN LA PROVINCIA DE BUENOS AIRES, ARGENTINA: COMPARACIÓN ENTRE DOS DISTRITOS ESCOLARES. UNA PERSPECTIVA NEO-INSTITUCIONALISTA
}

\author{
M. Fernanda Astiz
}

RESUMEN: El presente artículo aporta evidencia empírica sobre políticas adoptadas, resultados y consecuencias de las iniciativas de descentralización y autonomía educativa implementadas en Argentina en los años noventa. Este estudio realiza una evaluación de las implicancias del concepto de autonomía educativa en el país y de cómo fue adoptado tanto a nivel provincial como en las escuelas. Utilizando un paradigma neo-institucionalista, y mediante el uso de fuentes de información fidedignas de la provincia de Buenos Aires y de dos distritos escolares del Gran Buenos Aires, el estudio analiza la función que los factores organizativos cumplen en la implementación de la reforma. El objetivo de la presente investigación es analizar en forma comparativa las características institucionales y las consecuencias que las mismas acarrean en distritos que atienden poblaciones sociopolíticas diferentes.

Palabras clave: Autonomía escolar; Buenos Aires, Argentina; Descentralización; Neo-institucionalismo; Contextos sociopolíticos.

\section{Educational autonomy in Buenos Aires, Argentina: A COMPARISON AMONG SCHOOL DISTRICTS. A NEO-INSTITUTIONALIST PERSPECTIVE}

ABSTRACT: This article provides evidence of how decentralization and school autonomy policies were adopted and adapted in Argentina during the 1990s. It also addresses the consequences of their implementation at the provincial and school levels. Applying a neo-institutional framework, the study analyzes the role that organizational factors play in the implementation of school autonomy reform across diverse sociopolitical environments in the province of Buenos Aires.

Keywords: School autonomy; Buenos Aires, Argentina; Decentralization; Neo-institutionalism; Socio-political environments.

\footnotetext{
* Canisius College, Departamento de Educación. Búfalo, Nueva York, Estados Unidos. E-mail de contacto: astizm@canisius.edu.
} 


\title{
Autonomie Éducative À Buenos Aires, Argentine: COMPARAISON ENTRE DEUX DISTRICTS SCOLAIRES. UNE PERSPECTIVE NÉO-INSTITUTIONNELLE
}

\begin{abstract}
RÉSUMÉ: Cet article fournit des données empiriques sur les politiques adoptées, les résultats et les conséquences des initiatives de décentralisation et l'autonomie éducative mis en œuvre en Argentine dans les années 1990. On a effectué une évaluation des implications du concept de l'autonomie pédagogique dans le pays et comment il a été adopté, à la fois dans le niveau provincial et dans les écoles. En utilisant un paradigme néo-institutionnaliste, et grâce à l'utilisation de sources d'informations fiables de Buenos Aires et des deux districts scolaires de sa région métropolitaine, l'étude examine le rôle des facteurs organisationnels dans la mise en ouvre de la réforme. Le but de cette recherche est d'analyser, de manière comparative, les caractéristiques institutionnelles et de ses conséquences pour les districts qui répondent à différentes populations sociopolitiques.
\end{abstract}

Mots-clés: L'autonomie de l'école; Buenos Aires, Argentine; Décentralisation; Néo-institutionnalisme; Contextes socio-politiques.

\section{Introducción ${ }^{1}$}

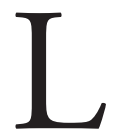

a autonomía educativa constituye una reforma impulsada en el marco de tendencias recientes a nivel mundial que se orientan hacia la descentralización. Esta reforma, basada en principios democráticos, tiene como objetivo fortalecer la efectividad educativa mediante la participación activa de la comunidad educativa, es decir, el cuerpo docente, los padres y los alumnos, en la administración escolar. La interpretación e implementación de esta reforma organizacional varía en forma considerable en las distintas regiones geográficas. Sin embargo, en la mayoría de los casos, se espera lograr dos objetivos principales, por un lado, la participación democrática en el ámbito educativo y, por el otro, la rendición de cuentas. En la década del noventa, no sólo en Latinoamérica sino también a nivel mundial, muchos países implementaron algún tipo de reforma en materia de autonomía educativa a fin de alcanzar los objetivos señalados, pero se conoce poco acerca de sus resultados.

La mayoría de los autores que han abordado los conceptos de descentralización, autonomía educativa y participación escolar en América Latina ha centrado el debate a nivel discursivo, estableciendo vínculos entre los modelos neoliberales y las políticas de reestructuración económica (p. ej. PAVIGLIANITTI, 1991; ARNOVE, 1997; TORRES; PUIGGROS, 1997), o bien se han interesado 
en demostrar 'qué funciona' para mejorar el desempeño de los alumnos. (KING; OZLER, 1998; MCEWAN; CARNOY, 1998; WINKLER; GERSHBERG, 2000) Otros autores se han ocupado únicamente de demostrar las desinteligencias entre la finalidad de las políticas y su aplicación práctica. (p.ej. PRAWDA, 1993; BRASLAVSKY, 1999; DUSSEL et al., 2000; GOROSTIAGA DERQUI, 2001) Finalmente, en menor medida, el resto de la bibliografía ha analizado los resultados de las reformas orientadas hacia la descentralización y la autonomía educativa a nivel subnacional y escolar. (p.ej. CIGLUTTI, 1993; MUNÍN, 1994; DUSSEL; THISTED, 1995; FULLER; RIVAROLA, 1998; RHOTEN, 2000) En general, los análisis efectuados en América Latina en materia de autonomía educativa no le han dado demasiada trascendencia a la función que desempeñan los factores organizacionales y sociopolíticos en la implementación de reformas ni a la implicancia de estos factores en los resultados de dicho proceso.

Por contexto sociopolítico se entiende el contexto socioeconómico y político que impera en cierto ámbito. De conformidad con el enfoque sociológico neo-institucional (p.ej. DIMAGGIO; POWELL, 1991) y la literatura sobre movimientos sociales (p.ej. TARROW; 1994), la cultura política es definida en el presente como el modo legítimo de hacer política en un contexto dado, las reglas de juego de cierta organización política. El objetivo del presente artículo es proporcionar evidencia empírica en relación con la implementación, los resultados y las consecuencias de la autonomía educativa en diferentes distritos de la provincia de Buenos Aires, Argentina. Este estudio analiza cómo se desarrolló el proceso de cambio a escala subnacional y escolar y se concentra en la función que desempeñan los factores organizacionales y sociopolíticos en la adopción de la autonomía educativa a nivel provincial; asimismo se analizan las implicancias de dichos factores en los resultados del proceso de reforma.

\section{Marco teórico}

El interés analítico de las corrientes neo-institucionalistas se focaliza en intentar dilucidar el rol que juegan las instituciones en la determinación de los procesos y resultados tanto políticos como sociales. Por instituciones entienden las reglas formales, procedimientos y normas, convenciones sociales, símbolos, ritos y costumbres a partir de los cuales los actores dan sentido al mundo que los rodea. Los argumentos neo-institucionalistas no ven al comportamiento como un agregado de acciones individuales, sino que son las instituciones las que estructuran, condicionan y dan sentido al comportamiento de los actores u organizaciones. (CLEMENS; COOK, 1999, p. 442; JEPPERSON, 1991, p. 145)

La teoría neo-institucionalista sociológica sostiene que un estado-nación adopta modelos de desarrollo que responden a paradigmas culturales globales 
comunes a todos los actores políticos. (MEYER et al., 1997) O sea, los modelos culturales internacionales establecen patrones normativos comunes, raramente cuestionados, que determinan no sólo lo que sería lo más apropiado, sino qué políticas se deberían seguir en determinadas circunstancias. (MEYER et al., 1997; MEYER; SCOTT, 1992; MEYER; 2000) Dichos modelos generan un isomorfismo de estructuras y políticas de las cuales los actores raramente pueden escapar.

Para esta corriente neo-institucional, los actores están motivados a adoptar determinadas prácticas por una preocupación de estatus. Es decir, en un intento por legitimarse ante sus pares internacionales (otros estados-nación, organizaciones internacionales, $\mathrm{u}$ otros colegas que adoptaron las supuestas medidas con anterioridad), los actores nacionales adoptan prácticas o normas que ya poseen reconocimiento global. De esta forma, los actores nacionales adquieren una dependencia epistemológica de otros actores, llámese grupo de expertos o "comunidades epistémicas", para desarrollar y demostrar la viabilidad normativa y la racionalidad de las políticas locales que ponen en práctica. (DiMAGGIO; POWELL, 1983, p. 151-152; RAMIREZ; SOYSAL; SHANAHAN, 1997; WEYLAND, 2005; ASTIZ, 2004; 2006a; DOBBIN; SIMMONS; GARRETT, 2007)

Ahora bien, cuando las políticas son delineadas en consonancia con lineamientos externos, es probable que se genere una divergencia entre el propósito de la política y los resultados alcanzados. (MEYER et al., 1997, p. 154) Así es como los modelos culturales mundiales podrían verse modificados en gran medida durante el proceso de adopción. A nivel nacional o provincial, algunos elementos externos son más fáciles de adoptar que otros e incluso algunos podrían generar divergencias o incompatibilidades con las estructuras organizacionales locales. (MEYER; ROWAN, 1977; MEYER; SCOTT, 1983; DiMAGGIO; POWELL, 1991; MEYER et al., 1997)

A fin de satisfacer los estándares mundiales, la adopción de nuevas estructuras educativas se efectúa con el apoyo de entidades profesionales claves en el contexto institucional. (DiMAGGIO; POWELL, 1991; MEYER et al., 1997) Sin embargo, dado que las reformas en el ámbito educativo suelen involucrar un alto grado de incertidumbre y no se vinculan en forma directa con los resultados escolares (MEYER; ROWAN, 1978), las organizaciones educativas podrían simplemente optar por abordar el proceso de reforma en forma simbólica. (RAMIREZ; RUBINSON, 1979) Dicha estrategia les permitiría conservar intactas sus actividades, no alterar aspectos organizativos y conservar su legitimidad. (MEYER; ROWAN, 1978)

Más aún, y complementando la visión sociológica con la vertiente neo-institucionalista política conocida también como de elección racional, el interés por adoptar esas reformas en forma simbólica, estaría relacionado con los comportamientos de los actores organizacionales. Dentro de esta corriente de pensamiento 
neo-institucionalista, las instituciones son entendidas como productos de la acción humana y, por lo tanto, los resultados políticos remiten al comportamiento de los actores en determinados contextos organizacionales quienes actuarían conforme a las siguientes premisas: 1 . se guían por una batería fija de preferencias y gustos, 2 . actúan en forma instrumental con el objetivo de maximizar el alcance de sus preferencias, 3. esto último lo logran a través de un premeditado cálculo estratégico. (HALL; TAYLOR, 1996; SCHARPF, 1997)

A pesar de que existen distintos enfoques dentro del neo-institucionalismo político, en su mayoría, los mismos centran sus análisis en los agentes y conciben a las organizaciones como restricciones a sus accionar o como producto de las actuaciones necesarias para satisfacer sus intereses. (SHEPSLE, 1989) Las organizaciones institucionales son definidas como las reglas de juego que, en combinación con los mecanismos para hacerlas cumplir, condicionan las opciones de los actores. Estas reglas incluyen las leyes estatales, políticas de organismos y las normas de grupos sociales, como los condicionamientos socioeconómicos de los contextos organizacionales. (INGLAM; CLAY, 2000)

Hannaway (1993, p. 148) sostiene que si bien un contexto institucional amplio podría explicar el motivo por el que las escuelas y las organizaciones escolares son similares, los escenarios políticos locales pueden explicar la magnitud de sus diferencias. En su artículo, la autora analiza los efectos que las presiones políticas y el nivel de centralización del sistema producen en las escuelas públicas de los distritos de los Estados Unidos. Los resultados de su investigación indican que "[...] cuanto mayor sea la presión existente en un contexto político determinado, las posibilidades de que el control sea ejercido por autoridades centrales y no locales aumentan [...]". En consecuencia, las tendencias orientadas a la descentralización podrían enfrentarse a mayores obstáculos en contextos políticos con altos niveles de presión ya sea política como socioeconómica. (McGINN; STREET, 1986; HANSON, 1989; ELMORE, 1993) Veremos entonces cómo estas ideas se aplican y resuelven en los casos estudiados.

\section{La autonomía educativa en Argentina: implementación de políticas}

A comienzos de los años noventa, el gobierno argentino profundizó el proceso de descentralización educativa que se había iniciado hacia fines del 1970 . (SENÉN GONZALEZ; ARANGO, 1996) A diferencia de intentos previos, las reformas más actuales fueron equiparadas con los procesos de democratización, sobre la base de la soberanía local y de una mayor respuesta a la necesidad de las diversas comunidades educativas. Durante los años noventa, el proceso de descentralización comprendió no sólo la delegación de la responsabilidad financiera de 
las instituciones secundarias y terciarias nacionales, tanto públicas como privadas subsidiadas, a los gobiernos provinciales y a la ciudad de Buenos Aires, sino también la concesión de ciertas facultades decisorias en relación con la administración y la prestación de servicios a los nuevos sistemas educativos provinciales y a las escuelas. $^{2}$ Sin embargo, la administración central conservó sus facultades decisorias a nivel de elaboración de políticas, coordinación y control del diseño del sistema nacional de educación. Entre las atribuciones que se retuvieron a escala nacional se encontraban la evaluación de la calidad educativa, los lineamientos principales de los contenidos curriculares, los programas de educación compensatoria y la asistencia técnica a las provincias. (ASTIZ, 2006b)

Además de la reforma curricular y la reestructuración del sistema educativo que introdujo la Ley Federal de Educación (1993) (LFE), se previó implementar una iniciativa reformista clave para mejorar la calidad de la educación. La LFE dispuso que la escuela y su comunidad, a saber, directivos, docentes, padres, alumnos, ex alumnos, personal administrativo y auxiliar de la docencia y organizaciones representativas (p. ej. gremios docentes o cooperadoras escolares que canalizan la participación de los padres) se ubican en el centro de dichas reformas (Ley Federal de Educación, Arts. 41, 42, 1993). En este sentido, la ley impulsaba un nuevo sistema de administración educativa que todas las escuelas debían aplicar a los efectos de promover principios institucionales y educativos democráticos.

Este nuevo modelo de administración escolar debería implementarse a través del desarrollo de un Proyecto Educativo Institucional [PEI] (LFE, Art. 41, 1993). El desarrollo del PEI constituye un elemento esencial en el proceso de reforma orientado hacia la autonomía educativa en Argentina. El objetivo del proyecto consiste en adaptar las directivas a escala nacional y provincial y los esquemas curriculares al ámbito educativo. La participación de actores de la comunidad educativa (excepto directivos y docentes) en el diseño del PEI se encuentra primordialmente orientada a apoyar y mejorar la calidad educativa. (ZONA EDUCATIVA, 1996) Sin embargo, de conformidad con la ley, la participación de la comunidad educativa no debería afectar el ejercicio de las responsabilidades directivas y docentes (Ley Federal de Educación, Art. 42, 1993).

En suma, durante los años noventa, siguiendo el modelo global de descentralización educativa, Argentina implementó una reforma estructural en materia educativa que llegó a las escuelas. En el diseńo del PEI se entiende que cada institución educativa debe trabajar en forma colegiada para el desarrollo de modelos pedagógicos y de administración innovadores que generen educación de calidad. Sin embargo, luego de más de una década de adopción de políticas aún hay interrogantes: ¿Cómo se implementó la autonomía educativa a nivel provincial? ¿Cuáles son los resultados producto de la reforma a nivel escolar? ¿Han afectado los resultados de la autonomía educativa la estructura organizativa provincial y 
local? Estos interrogantes siguen pendientes en el sistema educativo argentino de allí el interés de este artículo.

\section{Diseño de la investigación y métodos}

La presente investigación adopta un enfoque metodológico cualitativo basado en el estudio de casos (YIN, 1994) y una estrategia de selección de casos críticos. (CRESWELL, 1998) Las unidades de análisis son dos distritos de la provincia de Buenos Aires, Argentina. El análisis se concentra en esta provincia por tres razones fundamentales. En primer lugar, Buenos Aires no sólo es una de las provincias más grandes de Argentina, concentrando el $38 \%$ de la población del país, sino que además es considerada su centro político y económico. (CONSEJO FEDERAL DE INVERSIONES, 1999) Sin embargo, al momento de haber realizado este estudio, la característica más saliente de la provincia era su economía quebrada. ${ }^{3}$ Otro punto importante a considerar es que la provincia de Buenos Aires instituyó desde sus orígenes un sistema educativo descentralizado de gobierno a nivel de los consejos escolares locales. (SOLARI, 1984)

Por último, la provincia se divide en 134 partidos o municipios que poseen autonomía política y administrativa. Cada partido constituye un distrito educativo. El Gran Buenos Aires (GBA), también conocido como el Conurbano bonaerense, se encuentra conformado específicamente por los partidos que rodean la ciudad de Buenos Aires. Aproximadamente dos tercios del total de la población educativa se concentra en esta región. (SITUACIÓN Y EVOLUCIÓN SOCIAL PROVINCIAL, 1998) Vicente López y La Matanza son los partidos que se analizan en el presente estudio y han sido seleccionados dadas las divergencias existentes entre sus respectivos contextos socioeconómicos.

El GBA está dividido principalmente en dos cordones de desarrollo demográfico e industrial. El primer cordón, que incluye a los partidos que lindan con la Ciudad Autónoma de Buenos Aires (CABA), fue el que se desarrolló primero. Vicente López es parte de ese cordón y se encuentra ubicado en la zona norte del GBA. Su desarrollo data hacia fines del siglo XIX y principios del XX durante el período de expansión de la elite y la clase media urbana a lo largo de la costa norte del Río de la Plata. Bordeando la costa se ubicaron astilleros, clubes náuticos, clubes de tenis, remo y rugby, entre otros. Vicente López no es sólo uno de los distritos más acomodados económicamente hablando, sino también uno de los más homogéneos. (FLOOD, 1991; KEELING, 1996) A su vez es uno de los municipios más pequeños del GBA, aunque con una alta densidad poblacional. Durante la década del noventa registró 8,576.7 habitantes por kilómetro cuadrado. (INDEC, Datos Censales, 1991) 
El segundo cordón, o cordón externo y más distante de la CABA, se desarrolló con posterioridad. En la zona oeste del GBA está ubicada La Matanza. Más del 50\% de su territorio se encuentra en el segundo cordón, a lo largo del Río Matanza. La margen nordeste del río está altamente urbanizada, concentrando más de un millón y medio de personas. (INDEC, Datos Censales, 1991) La parte suroeste, ubicada fundamentalmente en el segundo cordón del GBA está, en comparación, escasamente poblada y posee amplias zonas abiertas sin desarrollar. Si bien La Matanza es el partido más extenso del conurbano bonaerense y el más poblado de toda la provincia, su densidad promedio no superaba para fines de los años noventa los 3471.1 habitantes por kilómetro cuadrado. Si bien el empleo industrial decreció durante el periodo analizado, La Matanza concentraba la mayor cantidad de establecimientos industriales de la provincia. Y a pesar de los datos oficiales, muchos de los mismos fueron cerrados o abandonados cuando, durante los noventa, la Argentina abrió ilimitadamente su economía. (<http//www.gba.gov. ar>; KEELING, 1996)

El distrito escolar de Vicente López contaba entonces con 12 escuelas públicas secundarias y 54 privadas. La tasa de cobertura escolar secundaria era del $75 \%$ y la de analfabetismo del $0.8 \%$. Su población poseía un nivel socioeconómico elevado y sólo el $4.7 \%$ de sus habitantes vivían debajo del nivel de pobreza. La Matanza por su lado, poseía 59 escuelas de enseñanza secundaria pública y 68 de enseñanza privada, su tasa de cobertura escolar secundaria era del $48.7 \%$ y la de analfabetismo del 3\%. Es importante destacar que el $21.6 \%$ de sus residentes vivía entonces debajo del nivel de pobreza. Mientras que en Vicente Lopez casi el $30 \%$ de la población había completado alguna forma de escolarización terciaria, en La Matanza esa cifra descendía al 8\%. En una región marcada por las fuertes desigualdades, las personas castigadas por la pobreza trepaban al 36\% de los residentes de este partido. Grandes concentraciones de viviendas muy precarias son vistas con frecuencia en esta zona del GBA. Considerado uno de los bolsones de pobreza más importantes de la provincia, los planes sociales provinciales eran moneda corriente. ${ }^{4}$

Durante los meses en que se llevó a cabo la investigación se realizaron dos procedimientos cualitativos que consistieron en análisis de contenidos y entrevistas semiestructuradas. Se entrevistaron a treinta y tres informantes claves, entre ellos, maestros, directivos, padres, funcionarios locales y provinciales y dirigentes políticos. Los individuos entrevistados fueron seleccionados en forma diversa, pero fundamentalmente a través de la técnica de la bola de nieve y según una base geográfica. Los relatos fueron analizados y clasificados según temáticas comunes y el nivel de análisis, a saber, provincial, municipal y escolar. Las narrativas de los entrevistados son identificadas con un código. Cada código se compone de la siguiente manera: las primeras tres letras indican el lugar donde se llevó a cabo la entrevista. Luego, se indica la función del entrevistado y finalmente, el número de entrevista. 


\section{Resultados: el proceso de adopción en la Provincia de Buenos Aires}

Durante el gobierno de Eduardo Duhalde (1991-1999) se implementó un proceso de reforma educativa en la Provincia de Buenos Aires. En el año 1995, dos ańos después de que el Congreso dictara la LFE, la legislatura provincial sancionó la Ley Provincial de Educación No 11.612 (LPE). El gobierno provincial adoptó la reforma federal de educación y la adecuó a fin de alcanzar los siguientes objetivos: equidad, descentralización, participación, solidaridad y justicia social. Sin lugar a dudas la reforma educativa provincial era consecuente con la función social que el gobernador le adjudicó a la educación y con el espíritu integral de su gestión. Los objetivos referidos debían ser alcanzados mediante la reorganización de la estructura provincial de gobierno, la ejecución de programas de acción social dirigidos a garantizar el acceso para todos y la participación del grupo familiar, la comunidad ampliamente entendida y las asociaciones docentes legalmente reconocidas en la educación bonaerense. (PROGRAMA DE DESARROLLO SOCIAL, 1994; PROGRAMA DE GOBIERNO, 1994)

La estructura del gobierno provincial fue reorganizada en base a una descentralización administrativa regional conformada por 16 jefaturas regionales para cada rama del sistema educativo [Educación General Básica (EGB) y polimodal], designadas por la Dirección General de Educación y Cultura (DGEC). ${ }^{5} \mathrm{La}$ DGEC, de rango ministerial, es el organismo responsable en materia de educación provincial. Las jefaturas regionales fiscalizan el funcionamiento de las secretarías de inspección a nivel distrital. Dichas secretarías, a través de los inspectores de distrito, tienen a su cargo la implementación de la reforma a nivel distrital y el manejo del trabajo administrativo en relación con aspectos técnicos y pedagógicos del sistema educativo provincial. A modo de ejemplo, recae sobre el organismo la responsabilidad de cubrir cargos docentes vacantes y proveer de datos estadísticos a la DGEC. (LPE, Art. 48, 1995)

A nivel distrital, los consejos escolares elegidos localmente, creados originalmente con el objeto de constituirse como unidades de participación política, se convirtieron en intermediarios administrativos entre la administración municipal y las cooperadoras escolares, fundamentalmente para movilizar los recursos para las escuelas dentro de cada distrito. Los consejos escolares locales no cuentan con recursos propios, pero están a cargo de viabilizar el financiamiento de los comedores escolares y el mantenimiento de la infraestructura escolar. Las distintas municipalidades, en un intento de adaptarse a la nueva organización provincial, crearon secretarías de cultura y educación que, en algunos casos, comenzaron a asumir un papel activo, aunque en el marco de una autoridad administrativa limitada. Las municipalidades tienen una participación insignificante en la prestación 
de servicios educativos. Los servicios que proveen son de educación inicial, infantil o parvularia. Sin embargo proporcionan fondos adicionales a las escuelas, que usualmente son destinados a obras de reparaciones de infraestructura y otros gastos menores. La mayoría de los fondos destinados a las escuelas públicas provienen de la DGEC. (EVOLUCIÓN GASTO PÚBLICO SOCIAL, 1997)

Las cooperadoras escolares constituyen fuentes adicionales de financiamiento. Cada escuela en la provincia cuenta con una cooperadora cuyos fondos se conforman con los aportes voluntarios de los padres. El monto de los aportes varía según los distritos y las condiciones socioeconómicas de las familias. En general, estos aportes no son suficientes para hacer frente a todas las erogaciones de la escuela y en consecuencia las cooperadoras organizan eventos para recaudar fondos o bien, intentan obtener aportes privados. Los fondos recolectados se utilizan con frecuencia para cubrir gastos escolares y actividades extracurriculares. Aunque se entienda que son instituciones participativas, las actividades de las cooperadoras no abarcan aspectos técnicos, administrativos o disciplinarios, excepto que la propia escuela así lo requiera (LPE, Art. 4, 1995).

En forma similar a la LFE, la ley provincial ubica a la unidad escolar en el centro del proceso de reformas (LPE, Art. 19, 1995). A diferencia de la LFE, la ley provincial promueve la participación de actores de la sociedad civil en la educación formal y no formal. Específicamente, alienta la concertación de convenios entre la DGEC y asociaciones intermedias para la realización de programas conjuntos de educación no formal (LPR, Arts. 3, 16, 1995). Dicho resultado sólo puede alcanzarse si existe una participación de la comunidad en general en la prestación de servicios educativos.

En la práctica la promoción de servicios se instrumentaba a través de un programa de acción social, desarrollada por las redes sociales del Partido Justicialista (PJ), que utilizaba la escuela como espacio para generar vínculos entre quienes brindaban dichos programas y sus beneficiarios. Tal como lo expresara un funcionario provincial, "Duhalde no pudo dejar de lado las prácticas populistas tradicionales al implementar políticas de tipo neoliberal; las necesitaba si quería ser reelecto en su cargo o si aspiraba a alcanzar la presidencia en el futuro" (EBAONo.2). Particularmente en este punto es donde se observa que la adaptación del principio de autonomía educativa adquiere características propias a nivel provincial. (ASTIZ, 2006b)

El 'Plan Social', como se denominó al programa de acción social, constituyó uno de los pilares del proceso de reestructuración educativa en la provincia. En un contexto de dificultades económicas, el programa tuvo manifestaciones diversas a nivel escolar, tales como planes alimentarios para las escuelas EGB y becas para alumnos del polimodal. Estos planes compensatorios se financiaron a través de canales alternativos que utilizaban fondos extraordinarios de transferencias 
nacionales y préstamos externos. La asignación de tales fondos era discrecional, como fue el caso del Fondo de Reparación Histórica. En vez de distribuirse según métodos ya preestablecidos (de la DGEC a los consejos escolares y finalmente a las escuelas), los fondos fueron transferidos directamente desde la administración provincial a las escuelas o a los beneficiarios en particular. (ASTIZ, 2004)

Tal como lo expresara una de las personas entrevistadas, 'los planes sociales constituían la conexión inmediata entre el gobierno, o más bien, Duhalde, y los pobres' (ELMPLNo.4); y fueron utilizados como un mecanismo de clientelismo político para vincular a las masas con el líder. La reforma en materia de autonomía educativa abrió el juego a la participación de la comunidad, aunque en un modo muy disímil al estipulado por la reforma legislativa nacional. Su implementación por otro lado dependió de las características del contexto sociopolítico en el que se desarrollaban.

\section{Vicente López}

Entre 1987 y 2011, el intendente Enrique García, de extracción política radical, ganó las elecciones locales de forma consecutiva con márgenes relativamente amplios. Ocupó, al momento que el estudio fue realizado, la intendencia de uno de los distritos más ricos del GBA por cuatro términos. García había construido su poder político a través de su liderazgo, carisma personal y, fundamentalmente, la notoria percepción de eficiencia de su gestión. Los diarios de circulación nacional han considerado el caso de este distrito como una municipalidad con una gestión exitosa. (LA NACIÓN, 2000) Tal vez, estos sean los motivos por los que los líderes educativos identificaron a Vicente López como un 'lugar excepcional'. (EVLSPNo.1) La imagen del intendente y sus cualidades de gestión le permitieron permanecer en el poder por más de 15 años y alzarse por encima de la fragmentación partidaria. Durante la gestión de García, la organización administrativa de Vicente López fue reestructurada con el objeto de satisfacer en forma eficiente las demandas sociales emergentes. De este modo, dentro de la administración municipal, se crearon varias secretarías que, en general, trabajan en colaboración con asociaciones no gubernamentales.

Dada la estrecha relación que García estableció con empresas locales, la administración municipal trabajó en forma conjunta con la Fundación Empresaria. Esta organización informal que provee fondos para el municipio fue creada en 1987 para subsidiar mejoras en el ámbito educativo, eventos culturales, y centros de salud locales. Las empresas locales que colaboran con la fundación obtienen exenciones impositivas. La Dirección General de Educación de Vicente López y el consejo escolar local, también una institución de base política radical, tuvieron a su cargo la asignación y distribución de los subsidios educativos. (EVLPANo.1) 
La administración municipal utiliza los subsidios a los efectos de establecer vínculos directos con la población electoral y para hacer frente a los fondos limitados que recibe de la administración provincial. Este último aspecto es atribuido a la rivalidad política entre la intendencia y la gobernación provincial. Precisamente, la deuda de la administración provincial con el municipio le confiere autonomía al intendente para diseñar su agenda política y, en forma simultánea, fortalecer su compromiso constante con Vicente López a través de un discurso cuidadosamente preparado que destaca la eficiencia de su gestión. (EVLCENo.3)

\section{La Matanza}

A lo largo del trabajo de campo desarrollado, las personas entrevistadas caracterizaron la situación de La Matanza como caótica. Según palabras de un líder educativo, "[...] como en el tango de Descépelo, un cambalache. Vivimos en una situación de anomia [...] un contexto muy difícil para enseñar y aprender”. (ELMTNo.1)

La Matanza ha sido históricamente un distrito liderado por el PJ. A diferencia de Vicente López, no ha sido gobernada por un único líder. Desde 1987, distintas líneas dentro del PJ se han alternado en el ejercicio del poder. En general, los intendentes de La Matanza pertenecieron a la misma línea interna del partido del gobernador. El PJ obtuvo triunfos políticos en La Matanza sobre la base de su 'organizada desorganización', caracterizada por una estructura informal descentralizada y compleja, conformada por una red asistencialista de asociaciones vecinales, punteros o mediadores políticos. (AUYERO, 2000; LEVITSKY, 2001; ASTIZ, 2002) Por medio de esta red, el PJ pudo mantener una práctica clientelar, en base a fondos gubernamentales y otros recursos no materiales: como alimentos, medicamentos, puestos de trabajo y contactos. (ELMPLNo.4)

Un funcionario local describió La Matanza, en razón de la complejidad e informalidad de su administración, de la siguiente manera:

Matanza equivale a pobreza, desempleo, programas sociales para aliviar la pobreza, clientelismo electoral, corrupción, protestas, frustración generalizada... tierra de nadie, simplemente, un caos. Es el espejo del justicialismo y la dinámica política más profunda de este partido. (ELMMONo.3)

Los actores escolares manifiestan que el consejo escolar local no es nada efectivo para satisfacer sus demandas, lo cual demuestra la ineficiencia anteriormente apuntada. (ELMSPNo.5) A pesar de que el PJ conservó su tradicional mayoría en el consejo escolar sus cargos se han diversificado desde 1999. Según 
un miembro de la legislatura local, “[...] esta circunstancia dificultó aún más la situación. Aunque aún conservamos la mayoría, hay mucha competencia entre las fuerzas, lo que retrasa las cosas todavía más [...]”. (ELMCENo.1) Los docentes hacen hincapié en que la educación nunca fue una prioridad en este distrito y que la educación en La Matanza nunca fue una prioridad para el gobierno provincial. Esta situación se evidencia en el hecho de que en 1999, el consejo escolar local declaró al municipio en estado de emergencia educativa. Algunos de los problemas identificados comprenden malas condiciones de los establecimientos educativos, falta de docentes y mobiliario básico, y contaminación del agua. (SITUACIÓN DE LAS ESCUELAS DEL DISTRITO, 1998-1999)

Por otro lado, en este distrito se informó la existencia de irregularidades en la distribución de programas sociales, lo que también genera dudas en cuanto a su eficacia. Los docentes y los directivos locales expresaron que, en muchos casos, algunas cooperadoras escolares son absorbidas por mediadores políticos, quienes utilizan la escuela como un centro para la distribución de planes sociales. (ELMSPNo.5)

\section{Resultados de la autonomía escolar: visión desde los distritos}

\section{Colaboración entre docentes y directivos}

Los resultados de los distritos son similares y por tales circunstancias se exponen en forma conjunta.

De conformidad con la LFE, las acciones de colaboración entre directivos y docentes constituyen un elemento fundamental para un diseño exitoso del PEI. En general, los directivos tienden a visualizar su participación con los docentes de manera positiva. Se consideran abiertos y comprensivos. Los directores manifiestan la necesidad de construir el consenso con los docentes, padres e inspectores. Sin embargo, entienden que las dificultades propias del proceso de selección docente limitan las acciones de participación y colaboración de estos en la unidad escolar. (EVLSPNo.3)

El equipo de conducción también indica que la celeridad y la falta de planificación que caracterizaron el proceso de reforma educativa, más la separación entre las ramas administrativas y pedagógicas y por área (p. ej. educación especial, educación física etc.) afectaron también la colaboración al interior de la escuela. Según manifiesta un director escolar:

La reforma incorporó $8^{\circ}$ y $9^{\circ}$ año a las antiguas escuelas primarias. En muchos casos, como la construcción de nuevas aulas en las instituciones educativas primarias se encuentra en ejecu- 
ción, octavo y noveno año funcionan como una unidad semi independiente dentro de las escuelas polimodales. Así, tenemos chicos y docentes de las antiguas escuelas primarias trabajando en nuestra escuela pero bajo reglas y objetivos distintos. Esta [situación] constituye una fuente de conflictos. (EVLSPNo.2)

El hecho de que el personal de la escuela pertenezca a diferentes niveles educativos constituye un impedimento importante para la colaboración en el ámbito escolar. Además, esta situación afecta la capacidad de liderazgo de los directores.

En general, los docentes señalan la existencia de problemas de comunicación con los directivos e inspectores. Estos últimos coinciden en este punto. El origen de este problema radica en el hecho de que los docentes trabajan en varias escuelas y en consecuencia no pertenecen a ninguna, por ello se los denomina 'maestros taxi.' En este sentido, aunque los docentes y los directores mencionan que el PEI se ha implementado en forma exitosa en sus escuelas, los docentes reconocen la importante función que los directores han tenido en el diseño del proyecto. (EVLTNo.2) Las personas entrevistadas indican que el director de escuela es una pieza central en toda la transformación educativa. (ELMTNo.3)

El mismo criterio resulta aplicable a la cooperación entre docentes. Los docentes expresan que el tiempo para interactuar con sus colegas es escaso. Los docentes se encuentran prácticamente solos en el desarrollo de sus actividades. Asimismo manifiestan que, en las esporádicas oportunidades en que efectivamente conversan entre sí, las conversaciones versan sobre temas administrativos y rara vez sobre cuestiones pedagógicas. (ELMTNo.8) Sin embargo, si consideramos las múltiples presiones bajo las que trabajan y los bajos salarios que perciben, sus quejas en relación con la reforma educativa han sido tenues. Es evidente que existe una suerte de resignación.

Cuando se consultó específicamente a los directores de escuela sobre cuánta autonomía y flexibilidad tienen luego de la implementación de las nuevas regulaciones, la respuesta más frecuente fue: “Tengo más responsabilidades, pero no más control sobre la escuela. Tengo que informar todo lo que hago y créame, todo necesita la firma de otra persona”. (ELMSPNo.6) Los directores no sólo están a cargo de los aspectos pedagógicos de la escuela, sino que también deben controlar el mantenimiento de la infraestructura escolar, lidiar con los concesionarios del comedor, controlar e informar la asistencia de los docentes, solicitar maestras suplentes y ejecutar otras tareas administrativas. A la vez, los directores indican que dicho cúmulo de responsabilidades vino acompañado de un mayor control por parte de parte de las unidades de inspección. (EVLSPNo.1)

Tanto los docentes como los directores perciben al modelo provincial como un sistema muy rígido $\mathrm{y}$, en su calidad de subordinados, deben cumplir 
órdenes, no cuestionarlas. Además, el único espacio en el que sienten que tienen la oportunidad de expresar sus opiniones es en el diseño del PEI. Los directivos escolares consideran que el PEI no sólo es una herramienta importante de gestión en la escuela, sino también un instrumento para mejorar la calidad educativa. (EVLTNo.8) Los directores coincidieron en que tienen flexibilidad en la toma de decisiones relativas a la cooperadora escolar.

Según un inspector de alto rango, la administración provincial ha comenzado a delegar más control en los directivos; sin embargo, los inspectores creen que aún hay un largo camino por recorrer: 'Las escuelas todavía dependen mucho de nosotros. Esto se debe a que venimos de un sistema con una organización muy jerárquica... pero queremos resolver la menor cantidad posible de cuestiones a este nivel administrativo'. (ELMINo.5)

\section{La participación de los padres}

En términos generales, los docentes y los directivos manifiestan que los padres no participan en las actividades escolares. Sin embargo, la percepción de los docentes en relación con esta situación varía según el distrito. Por este motivo, en esta ocasión, los resultados se exponen en forma separada.

En Vicente López, docentes y directores señalan que los niveles de participación de la población han disminuido durante la última década y las escuelas no están al margen de tal tendencia.

La democracia en 1980 involucraba a todos... Hoy en día, la situación es distinta... Hacia inicios de los ańos noventa, las escuelas públicas de Buenos Aires experimentaron una suerte de vaciamiento, ya que la clase media prefirió enviar a sus hijos a instituciones privadas. Hoy, como consecuencia de las recientes dificultades económicas, las familias de clase media ya no pueden pagar una escuela privada y se ven obligados a mandar a sus hijos nuevamente a las escuelas públicas con resignación, no con convicción, como sucedía anteriormente. Esta situación afectó su participación. Además, si ambos padres trabajan, el tiempo para participar en las actividades escolares es escaso. (EVLSPNo.1)

Debido a la calidad en la enseñanza, las antiguas escuelas secundarias nacionales tenían una reputación muy alta en la sociedad. Hacia fines de los ochenta, la crisis económica y la gran cantidad de paros docentes debilitaron dicha percepción. En consecuencia, dentro de lo posible, los padres preferían enviar a sus hijos a instituciones privadas, tal como lo ilustra el relato del directivo escolar 
que antecede. Es importante mencionar que, durante estos ańos, la cantidad de escuelas privadas aumentó en forma considerable en el distrito; del total de 156 escuelas 119 son privadas. (DIRECCIÓN DE INFORMACIÓN Y PLANEAMIENTO EDUCATIVO, 2001)

La información indica que, si bien los padres participan, su intervención en la cooperadora escolar es limitada. En ciertos casos, la participación consiste, únicamente, en una contribución monetaria mensual, que también ha disminuido con los años. Los docentes y el equipo de conducción informan que, en promedio, cinco de cada diez padres participan en forma activa en la cooperadora. (EVLSPNo.3) De todos modos, ese porcentaje puede incrementarse en ocasión de ciertos eventos especiales que, en general, son organizados por el equipo de conducción escolar. Se observó que tanto el equipo de conducción como los docentes son compasivos con la difícil situación que hoy atraviesan muchos padres y en muchos casos justifican su falta de participación. Los directivos escolares señalaron que los niveles de participación también dependen de las características de la comunidad y del equipo de conducción: "[...] hay escuelas en las que los directores saben cómo integrar a los padres. Son más permisivos... y abiertos a sus demandas [...]". (EVLCENo.3)

En La Matanza las entrevistas denotan que, en general, la participación de los padres en las escuelas prácticamente no existe ya que está muy determinada por el nivel socioeconómico barrial. Los docentes y equipos de conducción sienten que las actitudes de los padres reflejan las bajas expectativas y lo poco que se involucran en la vida escolar de sus hijos. Aun así, en sus relatos resaltan que la población a la que la escuela brinda servicios no sólo presenta necesidades económicas e intelectuales, sino también emocionales. La comunidad educativa, sin lugar a dudas, siente que no se encuentra preparada para responder a esta suerte de enfermedad social. En consecuencia, ante esta situación socioeconómica extrema, el interés por interactuar con los padres es escaso, o se da en forma selectiva. (ELMCSNo.15)

Los crecientes niveles de pobreza en el distrito condicionan no sólo la participación de los padres en la escuela, sino también sus aportes a la cooperadora escolar. Dichos aportes constituyen un elemento fundamental para el funcionamiento de la escuela $y$, al ser insuficiente, los docentes deben disponer de sus propios recursos para adquirir útiles escolares. (ELMTNo.8) En este sentido, los docentes expresan que la falta de participación de los padres los obliga a involucrarse en la cooperadora escolar, situación que excede sus obligaciones. (ELMTNo.2)

Los docentes y el equipo de conducción reprochan la falta de compromiso de los padres y se refieren a ellos en forma despectiva. La relación entre el personal escolar y los padres, como así también la falta de confianza y la descali- 
ficación hacia los padres pueden ser algunas de las razones que alejen a estos de la escuela y de la educación formal de sus hijos. Los padres se sienten estigmatizados y disconformes con el trato que reciben y consideran que las posibilidades de que su participación marque alguna diferencia en el ámbito escolar son ínfimas. (ELMAPNo.2)

\section{Participación de la comunidad educativa en los diferentes distritos}

En Vicente López la información obtenida reveló que, con frecuencia, la escuela organiza anualmente dos o tres actividades abiertas a la comunidad. En general, estas actividades consisten en festivales para recaudar fondos. Otras actividades incluyen las ferias de ciencias, es decir, muestras escolares en las que se exhiben las capacidades de los alumnos a través de stands donde se trabaja en forma interactiva. A veces, la estructura edilicia de las escuelas es utilizada para eventos que organizan la municipalidad u organizaciones no gubernamentales (ONGs) a fin de educar a la población en materia de salud y profilaxis. Sin embargo, las escuelas no participan de la organización de tales eventos.

El consejo educativo y los inspectores escolares coordinan muchas de las actividades que la municipalidad y las ONGs organizan a nivel escolar. Los directores mencionaron que trabajan en conjunto con centros municipales tales como el Centro Municipal de la Nińez y Adolescencia y el Centro Municipal de la Mujer. Ambas son instituciones gubernamentales que prestan servicios en colaboración con las instituciones educativas locales, ofreciendo programas de atención psicológica y familiar y programas para el tratamiento de adicciones.

Las ONGs con cobertura nacional e internacional dan cuenta de una participación moderada en el ámbito educativo. Por ejemplo, Caritas, una organización católica, brinda en forma gratuita programas de tutorías en matemática, castellano y programas de apoyo escolar. El Rotary Club es una de las instituciones más activas en este municipio. Aporta medallas y premios para torneos intercolegiales de atletismo y becas de un año para alumnos en escuelas públicas. Otras fundaciones, como la Fundación BankBoston, Creedicoop, Conciencias, Poder Ciudadano, Fundación Citybank, Fundación grupo Clarín y Fundación diario La Nación han organizado actividades para escuelas de los niveles EGB y polimodal. Las escuelas privadas son las que registran mayores niveles de participación en dichas actividades. Muchas de las actividades consisten en competencias deportivas a nivel regional y nacional, pasantías, proyectos de instrucción cívica, etc. Las escuelas públicas que a veces hacen uso de los clubes de barrio para desarrollar actividades de educación física o eventos especiales, lo hacen sin cargo alguno.

En La Matanza los niveles de colaboración entre la escuela y la comunidad son bajos, excepto por las organizaciones comunitarias recientemente creadas 
a cargo de las unidades básicas o manzaneras locales. ${ }^{6}$ El relato de un director de escuela da cuenta de esta situación.

En el partido de la Matanza [segundo cordón] apenas si podemos cumplir con la responsabilidad primaria que tiene la escuela, es decir, enseñar. Nos resultaría imposible concentrarnos en algo más. Además, no podemos organizar actividades abiertas a la comunidad... si no, tendríamos a la unidad básica controlando la escuela. (ELMSPNo.11)

Los inspectores distritales informan que la comunidad educativa colabora más en las localidades más ricas o del centro del distrito [primer cordón] donde la escuela organiza actividades abiertas a la comunidad: festivales, ferias, exhibiciones. En algunos casos, las asociaciones vecinales prestan sus gimnasios para eventos de este tipo que las escuelas organizan para recaudar fondos. Además, directores e inspectores indican que las escuelas y los centros de salud municipales trabajan en forma conjunta en programas de educación para la salud y campañas de vacunación. Las escuelas técnicas, como parte de su modelo curricular, organizan pasantías en empresas locales.

Los funcionarios provinciales denuncian la escasa colaboración entre el consejo escolar local, la administración municipal y las unidades de inspección educativa regional para la resolución de las dificultades educativas del distrito. La interacción entre estas dependencias administrativas locales y provinciales se encuentra sujeta a qué línea del Peronismo gobierna en el municipio. Empero, tal como lo indica un inspector de alto rango, la situación se modificó desde la última administración municipal. "Ahora podemos sentarnos a discutir cuestiones educativas con el intendente [...]". (ELMPANo.6) Recientemente, a través del Plan Trabajar, patrocinado por el gobierno nacional, el gobierno municipal distribuyó subsidios por desempleo. Los beneficiarios de tales subsidios fueron empleados, por ejemplo, como encargados o en tareas de mantenimiento de la escuela. (ELMPANo.6)

De los relatos de los inspectores escolares y los equipos de conducción se desprende que estos abordan la colaboración de la comunidad con los aspectos financieros o asistencialistas. Es probable que la difícil situación por la que atravesaba el distrito los haya desalentado a considerar otros enfoques más innovadores en relación con las actividades extracurriculares o la toma de decisiones en la escuela.

Es factible mencionar que, en este distrito, a diferencia de lo que sucede en Vicente López, los actores no identificaron ni ONGs ni fundaciones privadas vinculadas con el desarrollo educativo. Esta circunstancia podría obedecer a que tales organizaciones no colaboran con las escuelas de La Matanza o bien, a que los 
actores no asocian dichas instituciones con la educación. Puede que este distrito, dadas sus condiciones sociopolíticas, no resulte atractivo para las fundaciones que buscan beneficiarse de su participación en actividades educativas. Además, las funciones que ciertas ONGs e instituciones gubernamentales desempeñan en Vicente López, son desarrolladas en La Matanza por asociaciones locales que forman parte de las redes sociales del partido justicialista. Es interesante destacar que las asociaciones comunitarias de La Matanza participan en actividades de educación no formal, lo que no resulta sorprendente dadas las precarias condiciones económicas y educativas imperantes en el distrito. Es probable que las personas y las familias busquen canales educativos alternativos para lograr una rápida inserción en el mercado laboral o bien, en otros casos, apoyo mediante tutorías para suplir la falta de ayuda escolar que las familias pueden brindar en sus hogares.

\section{Conclusiones}

El presente artículo analiza los efectos que los contextos sociopolíticos han tenido en la adecuación de las directivas nacionales en materia de autonomía educativa por parte del gobierno provincial. Aunque las directivas contienen preceptos generales producto de las tendencias globales en torno a este tema, los datos presentados advierten de cómo la administración provincial encontró alternativas para adecuar los recursos administrativos y financieros destinados al ámbito educativo conforme a las aspiraciones políticas de sus líderes, lo que significa implementar en forma simbólica el mandato general, sin alterar la organización. O sea, si bien se adoptan las políticas mundialmente aceptadas como válidas y proveyendo así legitimidad al cambio educativo (MEYER; ROWAN, 1978; RAMIREZ; RUBINSON, 1979), simultáneamente se observa una desviación de dichas normas culturales globales en los procesos de implementación. La explicación a las adaptaciones que se vislumbran como resultado de este estudio ha de encontrarse en las instituciones organizativas de los sistemas sociopolíticos que tienden a privilegiar ciertos intereses y desmovilizar otros. (HALL; TAYLOR, 1996)

La reciente descentralización y los intentos de reforma educativa fueron diseñados a nivel nacional y provincial bajo la premisa de que, en primer lugar, se inicia el proceso de reforma y dicho proceso conduce, en una segunda instancia, a criterios de implementación y resultados uniformes. Pese a dichas expectativas, los resultados muestran que no se puede dar por sentada esta premisa. Las políticas orientadas a descentralizar el sistema y a generar mecanismos de mayor responsabilidad en la educación argentina pueden resultar relativamente exitosas en algunos contextos, pero fracasar por completo en otros. (ELMORE, 1993; HANNAWAY, 1993; HANSON, 1997) El concepto que subyace es que, dentro 
de las características generales de la sociedad, los contextos sociopolíticos siguen determinando las reglas de juego, lo que genera acciones de implementación mixtas. (McGINN, 1996; ASTIZ et al., 2002)

Según las narrativas presentadas, la identidad sociopolítica parecería haber afectado los criterios de distribución de responsabilidades, influencias y participación de la comunidad educativa en las escuelas de estos dos distritos de la provincia de Buenos Aires, lo que trajo aparejado resultados divergentes. La información obtenida muestra que la participación y las relaciones entre los distintos sectores de la comunidad educativa varían entre los distritos. El comportamiento de cada actor es una respuesta óptima al comportamiento de los otros actores y a la estructura institucional existente y al cálculo estratégico de dichos actores políticos. (TSEBELIS, 1990)

Vicente López ha sido siempre un distrito con una economía próspera y, desde los inicios de los años ochenta, un territorio con cierta estabilidad política. Desde 1987, gracias a un fuerte liderazgo, la prosperidad le permitió al distrito no sólo desarrollar sus capacidades de gestión a través de secretarías y organizaciones locales, tales como la Fundación Empresaria, sino también manejar en forma satisfactoria la rivalidad política con la administración provincial y, en forma simultánea, ganar autonomía. Como el distrito efectivamente logró mayores niveles de autonomía, el intendente tuvo una concepción más amplia en relación con discursos globales sobre eficiencia y responsabilidad, elemento que también utilizó con fines electorales. La eficiencia de la administración municipal generó una red de colaboración relativamente exitosa entre los consejos escolares locales, el gobierno municipal, las inspecciones, las escuelas y las organizaciones locales. En Vicente López, la colaboración entre el gobierno y las asociaciones locales se presenta como un modo atractivo de revitalizar la educación, ya que ha facilitado la adaptación a las nuevas demandas educativas y a la ineficiencia de la administración provincial.

Resulta interesante señalar que esta revitalización no fortaleció las prácticas participativas dentro de las escuelas que, en este aspecto, se vieron mayormente influenciadas por la organización administrativa provincial. Docentes y directores rara vez compartieron experiencias, intercambiaron ideas acerca de la enseñanza o colaboraron entre sí en el proceso de instrucción. Las escuelas en las que se observaron estas características, si bien son una minoría, gozaron de una autonomía de facto en el pasado. ${ }^{7}$ El estudio revela la existencia de una paradoja entre las recientes reformas en materia de descentralización y autonomía educativa: las escuelas que participaban de un sistema de administración centralizada pero que gozaban de una autonomía de facto, vieron limitada dicha autonomía luego de que la reforma en materia de descentralización se efectivizó. Esta autonomía limitada se deriva del sistema organizacional en el que operan. (BRASLAVSKY, 1998) 
Inversamente, en La Matanza en donde los informantes dan cuenta de un modelo administrativo caótico, dicha organización si bien no implementó la reforma de la forma esperada, sus prácticas fueron favorables a los fines más inmediatos del distrito y del Partido Justicialista. En este caso, la organización del liderazgo del partido y la competencia interna no fomentaron ni la colaboración entre las diferentes unidades administrativas locales, el consejo escolar local, la administración municipal y la inspección, ni tampoco entre la escuela y las asociaciones barriales tradicionales. Sin embargo, la organización vinculó a la escuela con la estructura partidaria local y con organizaciones comunitarias constituyéndola en un canal para el desarrollo del patronazgo y, al mismo tiempo, un mecanismo de apoyo a la maquinaria justicialista local. (AUYERO, 2000)

La falta de colaboración al interior de la escuela en estos distritos puede obedecer a la concentración de poder en manos de la administración provincial en lo concerniente a prácticamente todos los aspectos de la vida escolar, lo que reduce las facultades decisorias del distrito y de las escuelas en relación con las actividades escolares cotidianas y las fuentes adicionales de financiamiento. (LAUGLO, 1996) Tal vez, la sobrecarga de tareas que pesa sobre los docentes y la inestabilidad laboral, como así también las excesivas exigencias que recaen sobre los equipos de conducción impiden la creación de vínculos de cooperación entre éstos. En ambos distritos las nuevas exigencias educativas han sido adaptadas con el objeto de conservar el statu quo. Esto es, las acciones implementadas no han fortalecido el discurso vigente en este tiempo: descentralización del poder y participación democrática. Más bien priorizaron reducir el margen de incertidumbre y las posibilidades de participación democrática de los actores involucrados. (SHEPSLE, 1989)

El análisis de la implementación de la autonomía educativa y sus resultados en la provincia de Buenos Aires ilustran que la autogestión escolar en un contexto de aplicación global de prácticas de descentralización, no siempre constituye un proceso universal, deseado y posible, tal como se lo presenta, ni tampoco contribuye en todos los casos al fortalecimiento de prácticas democráticas en la educación y en la sociedad civil. Por el contrario, constituye un proceso complejo que involucra diferentes sectores del estado y de la sociedad y depende de un aún más complejo grupo de factores organizacionales y sociopolíticos. (WEILER, 1993; INGRAM; CLAY, 2000; ASTIZ, 2006a) En este caso, el modelo explicativo que se nutre de la integración del NIS y el NIR apunta a realzar el valor de la relativa autonomía de los actores individuales y colectivos en el diseño, adaptación e implementación de políticas públicas de origen global. Los resultados de las políticas aplicadas ponen de manifiesto la complejidad de las percepciones que se asientan en múltiples inquietudes respecto de intereses políticos, choques culturales y tensiones a nivel municipal-provincial. (ASTIZ 2006b) 
El desafío que enfrentan las políticas educativas y la educación comparada se centra en la realización de investigaciones que exploren el complejo esquema de interacciones entre los contextos organizacionales y sociopolíticos que influyen en el proceso de delineación de políticas, su aplicación y consecuencias. Además, deberían centrarse en examinar cómo y en qué medida aspectos de carácter socio-económico determinan la aplicación e impacto de dichas políticas. Investigaciones de ese tipo deberán ahondar con mayor especificidad en la explicación de procesos y modelos imperantes en las distintas zonas geográficas a través de la utilización de paradigmas teóricos complementarios. Los resultados obtenidos de este tipo de enfoques seguramente facilitarán el diseño de políticas y la implementación de estrategias que se adapten a la realidad local.

\section{Notas}

1. La autora le agradece al Profesor Antoni Verger por su invitación a participar de este monográfico y las sugerencias provistas para modificar la versión final de este artículo. Se agradecen también los comentarios brindados por los evaluadores y editores de la revista para mejorar la calidad del manuscrito para su publicación. Por último, esta investigación no hubiera sido posible sin la participación de todas las personas entrevistadas en el marco del trabajo de campo.

2. Ver Ley de Transferencia de los Servicios Educativos N. 24.049 (1992); Ley Federal de Educación N 24.195 (1993).

3. Buenos Aires concentraba en su momento el $33 \%$ de la deuda total de las provincias y un índice de pobreza era del $35.3 \%$ (resultados parciales del Censo 2001, publicado en Clarín, Buenos Aires, 27 de agosto de 2001).

4. La información provista para cada municipio proviene del Instituto Nacional de Estadísticas y Censos (INDEC). Dirección Provincial de Estadística y Planificación General. Situación y Evolución Social Provincial, Buenos Aires. Síntesis N0.1. 1998.

5. La ley sancionada en 1993 también introdujo una nueva organización escolar que se componía de tres niveles, a saber, un año de educación inicial obligatoria, nueve años de educación general básica (EGB) obligatoria, y en forma opcional, la educación polimodal, de tres años de duración como mínimo. El sistema educativo argentino fue nuevamente modificado en el 2006.

6. Las manzaneras constituyen redes de mujeres organizadas. Se trata de líderes de la comunidad o intermediarias políticas locales. Unidad básica es el nombre de las sedes locales del Peronismo.

7. Con excepción de los inspectores nacionales, no existían otras unidades administrativas entre el ministerio y las escuelas. Dada la distancia geográfica entre la administración central y la escuela, como así también, la baja proporción de inspectores en relación con la cantidad de escuelas, las escuelas nacionales alcanzaron un mayor grado de autonomía educativa. De conformidad con las normas dictadas a nivel nacional, los directores de dichas escuelas secundarias estaban facultados para elegir su cuerpo docente. Además, la mayoría de los docentes concentraban todas sus horas cátedra en la misma escuela, cuestión que era poco frecuente en el sistema provincial. 


\section{Referencias bibliográficas}

ARNOVE, R. Neoliberal education policies in Latin America: arguments in favor and against. In: TORRES, C.; PUIGGROS, A. (Eds.). Latin American education. Boulder, CO: Westview Press, 1997, p. 79-100.

ASTIZ, M. F. Decentralization and educational reform. What accounts for a decoupling between policy purpose and practice? Evidence from Buenos Aires, Argentina. Public Administration and Management an Interactive Journal, v. 9, n. 2, 2004, p. 137-165.

. Policy enactment and adaptation of community participation in education: The case of Argentina. In: BAKER, D. P.; WISEMAN, A. W. (Eds.). The Impact of Comparative Research on Institutional Theory. San Diego, CA: Elsevier Ldt., 2006a, p. 305-332.

School autonomy in the province of Buenos Aires, Argentina: evidence from two school districts. Comparative Education, v. 42, n. 02, 2006b, p. 203-223.

ASTIZ, M. F.; WISEMAN, A.; BAKER, D. Slouching towards decentralization. Consequences of globalization for curricular control in national education systems. Comparative Education Review, v. 4, 2002, p. 66-88. [Special Issue on Globalization].

AUYERO, J. The logic of clientelism in Argentina: an ethnographic account. Latin American Research Review, v. 35, n. 3, 2000, p. 55-81.

BRASLAVSKY, C. Restructuring the Argentine educational system, 1984-1995. In: TULCHIN, J. S.; GARLAND, A. M. (Eds.). Argentina: the challenges of modernization. Wilmington, DE: Scholarly Resources, 1998, p. 297-313.

. Estudios de casos sobre la reforma de la gestión en América Latina. In:

(Ed.). La gestión en Busca del Sujeto. Santiago de Chile: UNESCO-Santiago, 1999, p. 70-92.

CIGLIUTTI, S. Los consejos de escuela: un estudio de casos sobre las políticas educativas de convocatoria a la participación en la gestión escolar. Propuesta Educativa, n. 5, 1993, 27-35.

CLARÍN. Datos Provisionales Censo 2001: la población de la Provincia de Buenos Aires supera los 13,7 millones de habitantes. 2001. Disponible en: <http://www.clarin.com/ diario/hoy/p-349354.htm>. Consultado el 27 de agosto de 2001.

CLEMENS, E. S.; COOK, J. M. Politics and institutionalism: explaining durability and change. Annual Review of Sociology, n. 25, 1999, p. 441-466.

CONSEJO FEDERAL DE INVERSIONES. Información de la Provincia de Buenos Aires. 1999. Disponible en: <http://www.cfired.org.ar/provin/s baires/f 1.htm>. Consultado el 7 de mayo de 2002.

CRESWELL, J. W. Qualitative inquiry and research design. Thousand Oaks, CA: Sage Publications, 1998.

DiMAGGIO, P. J.; POWELL, W. Introducción. In: (Eds.). The new institutionalism in organizational análisis. Chicago, IL: University of Chicago Press, 1991, p. 1-38. 
DIRECCIÓN DE INFORMACIÓN Y PLANEAMIENTO EDUCATIVO. Centro de Documentación e Información, Consulta de Datos de Establecimientos de la DGCyE, Distrito: V López. 2001. Disponible en: <http://abc.gov.ar/Escuelas/consultas/Establecimiento/establecimiento2.cfm>. Consultado el 30 de agosto de 2001.

DIRECCIÓN GENERAL DE CULTURA Y EDUCACIÓN. Ley Provincial de Educación No. 11.612, Provincia de Buenos Aires, 1995.

DOBBIN, F.; SIMMONS, B.; GARRETT, G. The global diffusion of public policies: Social construction, coercion, competition, or learning? Annual Review of Sociology, n. 33, 2007, p. 449-472.

DUSSEL, I.; THISTED, S. La descentralización educativa: el caso del municipio de San Fernando, provincia de Buenos Aires. Serie de Documentos e Informes de Investigación. Buenos Aires: FLACSO. 1995.

DUSSEL, I.; TIRAMONTI, G.; BIRGIN, A. Decentralization and re-centralization in the Argentine educational reform: reshaping educational policies in the 1990s. In: POPKEWITZ, T. (Ed.). Educational knowledge. Changing the relationships between the state, civil society, and the educational community. Albany, NY: State University of New York Press, 2000, p. 304-345.

ELMORE, R. School decentralization: who gains? Who loses? In: HANNAWAY, J.; CARNOY, M. (Eds.). Decentralization and school improvement. San Francisco, CA: Jossey-Bass, 1993, p. 33-54.

FISZBEIN, A. Institutions, service delivery and social exclusion: a case study of the education sector in Buenos Aires. Washington, DC: World Bank, 1999 [Paper Series n. 47].

FLOOD, C. A. Agricultura suburbana en pequeña escala en el Gran Buenos Aires. IFDA Dossier 81, 1991, p. 39-50.

FULLER, B.; RIVAROLA, M. Nicaraguas's experiment to decentralize schools: views of parents, teachers, and directors. Washington, DC: World Bank, 1998.

GOROSTIAGA-DERQUI, J. Educational decentralization policies in Argentina and Brazil: exploring the new trends. Journal of Education Policy, n. 16, v. 6, 2001, p. 561-583.

HALL, P. A.; TAYLOR, R. C. R. Political Science and the three new institutionalisms. Political Studies, n. 44, 1996, p. 936-957.

HANNAWAY, J. Political pressures and decentralization in institutional organizations: the case of school districts. Sociology of Education, n. 66, 1993, p. 147-163.

HANSON, M. Decentralization and rationalization in educational administration: comparison of Venezuela, Colombia and Spain. Comparative Education, n. 25, 1989, p. 41-55.

- Educational decentralization: issues and challenges. Santiago, Partnership for Educational Revitalization in the Americas, Occasional Paper No. 9, 1997.

INDEC. Censo 1991. Ministerio de Economía, Obras y Servicios Públicos. Buenos Aires, Argentina, 1991. . Encuesta Permanente de Hogares. Buenos Aires: INDEC, oct. 1990-1998. 
INDEC. Evolución Gasto Público Social. Buenos Aires: Dirección Nacional de Programación del Gasto Social. Secretaría de Programación Económica y Regional, 1997.

INGRAM, P; CLAY, K. The choice-within-constrains new institutionalism and implications for sociology. Annual Review of Sociology, n. 26, 2000, p. 525-46.

JEPPERSON, R. L. Institutions, institutional effects, and institutionalism. In: POWELL, W. W.; DIMAGgIO, P. J. (Eds.). The New Institutionalism in Organizational Analysis, Chicago, IL: University of Chicago Press, 1991, p.143-163.

KEELING D. Buenos Aires. Global dreams, local crises. West Sussex: John Wiley \& Sons Ltd., 1996.

KING, E.; OZLER, B. What's decentralization got to do with learning? The case of Nicaragua's school autonomy reform. Washington, DC: World Bank, 1998.

LA NACIÓN. El gobierno municipal, más cerca de los ciudadanos. Estar junto a la gente, Buenos Aires, 6 jul. 2000.

LAUGLO, J. Forms of decentralization and their implications for education. In: CHAPMAN, J. D. et al. (Eds.). The reconstruction of education. Quality, equity and control. London: Cassell, 1996, p. 19-46.

LEVITSKY, S. An 'organized disorganization': informal organization and the persistence of local party structures in Argentine Peronism. Journal of Latin American Studies, n. 33, 2001, p. 29-65.

LEY FEDERAL DE EDUCACIÓN no. 24195, 1993. Disponible en: < http://www. mcye.gov.ar>. Consultada en 28 de agosto de 1997.

MARCH, J.; OLSEN, J. The new institutionalism: Organizational factors in political life. American Political Science Review, n. 88, 1984, p. 734-749.

McEWAN, P.; CARNOY, M. Public investments or private schools? A reconstruction of educational improvements in Chile. Washington, DC: World Bank, 1998.

McGINN, N. Education, democratization, and globalization: a challenge for comparative education. Comparative Education Review, n. 40, 1996, p. 341-357.

McGINN, N.; STREET, S. Educational decentralization: weak state or strong state? Comparative Education Review, n. 30, 1986, p. 471-490.

MEYER, J.; ROWAN, B. The structure of educational organizations. In: MEYER, J. (Ed.). Environment and organizations. San Francisco, CA: Jossey-Bass, 1978.

MEYER, J. W. et al. World society and the nation state. American Journal of Sociology, n. 103,1997 , p. 144-181.

MEYER, J. W.; SCOTT, W. R. (Eds.). Organizational environments: ritual and rationality. Beverly Hills, CA: Sage Publishers, 1983.

MINISTERIO DE ECONOMÍA OBRAS Y SERVICIOS PÚBLICOS. Ley de Transferencia de los Servicios Educativos No. 22.049, Buenos Aires, 1992. 
MUNÍN, H. ¿Más poder para la administración local? Una perspectiva crítica en torno a la descentralización en la provincia de Buenos Aires, Argentina. Revista Latinoamericana de Estudios Educativos, n. 24, 1994, p. 83-106.

PAVIGLIANITTI, N. Neo-conservadurismo y educación. Buenos Aires: Libros del Quirquincho, 1991.

PRAWDA, J. Educational decentralization Latin America: lessons learned. International Journal of Educational Development, n. 13, 1993, p. 253-264.

PROGRAMA DE DESARROLLO SOCIAL. Gobernación Duhalde. 1994. Disponible en: <http://www.gobernacionduhalde.com.ar/cgi-bin/framed/3365/obras/consejos/consejfam.html>. Consultado el 19 de abril de 2002.

PROGRAMA DE GOBIERNO. Dirección de Educación y Cultura de la Provincia. 1994. Disponible en: <http://www.gobernacionduhalde.com.ar>. Consultado el 19 de abril de 2002.

RAMIREZ, F.; RUBINSON, R. Creating members: the political incorporation and expansion of public education. In: MEYER, J.; HANNAN, M. (Eds.). National development and the world system. Chicago, IL: University of Chicago Press, 1979.

RAMIREZ, F. O.; SOYSAL, Y.; SHANAHAN, S. The changing logic of political citizenship: Cross-national acquisition of women's suffrage rights, 1890 to 1990 . American Sociological Review, n. 62, 1997, p. 735-745.

RHOTEN, D. Education decentralization in Argentina: a 'global-local conditions of possibility' approach to state, market, and society change. Journal of Education Policy, n. 15, 2000, p. 593-619.

SCHARPF, F. W. Games Real Actors Play: Actor-Centered Institutionalism in Policy Research. Boulder, CO: Westview Press, 1997.

SENÉN-GONZÁLEZ, S.; ARANGO, A. La descentralización en la reforma educativa Argentina. ¿Politica educativa o politica fiscal? Paper distribuido en el Congreso Internamericano del CLAD sobre la reforma del estado y la administración pública, Rio de Janeiro, 7 de nov. 1996.

SHEPSLE, K. Study institutions: Some lessons from the rational choice approach. Journal of Theoretical Politics, v. 1, n. 2, 1989, p. 131-149.

SOLARI, M. Historia de la educación argentina. Buenos Aires: Editorial Paidós, 1984.

TARROW, S. Power in movement: social movements, collective action and politics. Cambridge: Cambridge University Press, 1994.

TORRES, C.; PUIGGROS, A. Latin American education. Boulder, CO: Westview Press, 1997.

TSEBELIS, G. Nested Games. Rational Choice in Comparative Politics. Berkeley: University of California Press, 1990. 
WEILER, H. Control versus legitimization: the politics of ambivalence. In: HANNAWAY, J.; CARNOY, M. (Eds.). Decentralization and school improvement: can we fulfill the promise? San Francisco, CA: Jossey-Bass, 1993.

WEYLAND, K. Theories of policy diffusion: Lessons from Latin American pension reform. World Politics, n. 57, 2005, p. 262-295.

WINKLER, D.; GERSHBERG, A. Los efectos de la descentralización del sistema educacional sobre la calidad de la educación en América Latina. Santiago de Chile, PREAL, Documento de Trabajo No.17, 2000.

YIN, R. Case study research: design and methods. Newbury Park, CA: Sage, 1994.

ZONA EDUCATIVA. Proyecto educativo institucional. 1998. Disponible en: <http://www.zona.mcye.gov.ar/zona98/ZonaEducativa/Revista11/Revista11html>. Consultado el 7 de mayo de 2002.

Recebido em 23 de julho de 2015.

Aprovado em 01 de setembro de 2015.

DOI: http://dx.doi.org/10.1590/ES0101-73302015152402 